\title{
The Influence of Infectious Diseases on Dentistry
}

\author{
${ }^{1}$ Raghunath Puttaiah, ${ }^{2}$ Mahesh Verma, ${ }^{3}$ Shankar Gouda Patil, ${ }^{4}$ Anil Reddy \\ ${ }^{1}$ Associate Professor, Department of Diagnostic Sciences, TAMUS HSC Baylor College of Dentistry, Dallas, Texas, USA \\ ${ }^{2}$ Director and Principal, Maulana Azad Institute of Dental Sciences, New Delhi, India \\ ${ }^{3}$ Assistant Professor, Department of Oral Pathology, KLE Society's Institute of Dental Sciences, Bengaluru, Karnataka, India \\ ${ }^{4}$ Faculty, Department of Pediatric Dentistry, TAMUS HSC Baylor College of Dentistry, Dallas, Texas, USA
}

Correspondence: Raghunath Puttaiah, Associate Professor, Department of Diagnostic Sciences, TAMUS HSC Baylor College of Dentistry, Dallas, Texas, USA, e-mail: puttaiah.raghunath@gmail.com

\section{ABSTRACT}

Dentistry, predominantly a surgical field with frequent exposure to blood and body fluids, is a high-risk occupation with respect to occupationally acquiring infectious diseases. On the same note, patients are also at risk of being infected, if adequate infection control measures are not strictly followed. Traditionally, based on the routes of disease transmission, we can categorize diseases that are bloodborne, airborne and also through fomites. Within these traditional categories also fall the new and emerging diseases that have had serious public health consequences of morbidity and mortality. As a health care provider, dentists must understand the impact of these diseases, and strictly implement practical disease control measures during provision of dental care and reduce the spread within the clinical arena. Common diseases of public health concern that need to be addressed are bloodborne diseases, such as hepatitis A, E, B, C, D and G, HIV; respiratory diseases such as tuberculosis, influenza, severe acute respiratory syndrome (SARS), AH1N1 influenza and immunizable childhood diseases. Apart from infection control measures, we must implement public health policy measures, such as immunization of current and prospective health care personnel (students in the dental profession) against immunizable diseases, utilize disease screening measures, postexposure disease control measures and utilize standard and additional precautions, the latter as required in certain instances.

Keywords: Infectious diseases, Bloodborne diseases (HIV, hepatitis B, C, D, G), Other hepatic diseases (hepatitis A and E), Respiratory diseases (tuberculosis, influenza, SARS, AH1N1 influenza), Immunizable childhood diseases, Public health measures, Public policy measures.

\section{INTRODUCTION}

Common infectious diseases impacting the community’s common conditions are different hepatitis-causing viruses, HIV and AIDS, and tuberculosis (TB) including multidrug-resistant TB. ${ }^{1-9}$ While hepatitis B, C, D and G viruses follow a bloodborne route of transmission, hepatitis $\mathrm{A}$ and $\mathrm{E}$ are transmitted through the fecal-oral route. Contamination of food and water through improper and unhygienic handling are associated with fecal-oral route of transmission for hepatitis A and $\mathrm{E}$, and seen in lesser developed regions of the world. HIV infection and tuberculosis occurring individually or as comorbidities are common conditions present in many regions of the world and in certain segments of the population even in medically advanced countries. ${ }^{10}$ Multidrug-resistant tuberculosis (MDR-TB) and extremely drug resistant tuberculosis are also a major concern. ${ }^{11}$ In the past decade, there have been epidemics of respiratory diseases and infections, severe acute respiratory syndrome (SARS) and AH1N1 influenza that have caused chaotic effects with respect to day-to-day living in most countries in the world. ${ }^{12,13}$ Other common infectious conditions, such as herpetic infections, influenza and bacterial infections may have an impact on the clinicians providing care if they are infected.

\section{Bloodborne Diseases}

Hepatitis A virus (HAV) belongs to the Hepatovirus genus and Picornaviridae family and is an RNA virus. ${ }^{14}$ Four genotypes affect humans, but there is only one serotype. HAV infection causes only acute inflammation of liver, mild to severe signs and symptoms including jaundice, but rarely resulting in death. Among otherwise healthy adults, the death rate resulting from HAV infection is about one in 1,000 , and in people over 50 years of age, the rate is 27 in 1,000 . The incubation period is about 4 to 6 weeks or about 15 to 50 days, where the patient is normally not symptomatic but is usually shedding viruses in the stool. Once a person recovers from hepatitis A infection, the person is protected for life against all strains of HAV. A serology of antiHAV means history of past infection. A vaccine against hepatitis A viral infection is now available in most countries and may be available as a stand-alone [Havrix (GlaxoSmithKline) or Vaqta (Merck)] or in combination with a vaccine for hepatitis B virus infection [Twinirix (GlaxoSmithKline)]. If one has not been exposed to HAV, a one-time vaccination may provide life-long immunity. All dental students that have not been immunized against or who have not been infected with HAV should be encouraged to get immunized.

Hepatitis $E$ virus $(\mathrm{HEV})^{15}$ is an RNA virus and can also spread through fecal-oral route. Hepatitis E viral infection is similar in 
nature to the HAV infection epidemiologically except for the higher rate of infection among pregnant women in the third trimester (20\% infection rate). Outbreaks are commonly seen in the South Asia, Southeast Asia, Africa, Central and South American regions among other geographic regions in the world. As of today, there is no vaccine available against hepatitis $\mathrm{E}$ virus.

Hepatitis B viral (HBV) infection is caused by a DNA virus, that is a hepadnavirus. Most patients with $\mathrm{HBV}$ infections cannot be clinically identified as being infected. About 2 to $7 \%$ of the population in Southern Asia, the Middle East, the Mediterranean, Eastern Europe, Russia and parts of Central and South America are infected with this virus. ${ }^{4,5,16}$ Certain regions in Alaska and Canada (the Tundra region), South America, Africa and Southeast Asia including China are considered high in prevalence ( $>8 \%$ of the population). Most of the regions in North America, parts of South America, Australia and Western Europe are considered low prevalence areas ( $<2 \%$ of the population).

The incubation period lasts from 45 to 160 days, therefore it is also called "chronic hepatitis infection”. Transmission can be both percutaneous and nonpercutaneous, but it is primarily bloodborne. This variety of hepatitis is very contagious and has been occupationally acquired by dentists in the past. Outcomes of HBV infection are: about $90 \%$ of the infected become healthy again; about 9 to $10 \%$ become asymptomatic carriers or suffer from chronic, persistent hepatitis or develop active hepatitis leading to hepatocellular carcinoma and death; about 1\% develop fulminant disease after infection and die.

Vaccines against HBV infections are available in most countries. In the past, the rate of infection among dentists (general practitioners and specialists included) has ranged from 13.6 to $38.5 \%$. Therefore, it is not an uncommon disease affecting dentists. There have been cases of dentists infecting patients with HBV. According to the Centers for Disease Control and Prevention (CDC), booster doses of the vaccine may not be necessary due to the anamnestic response and lack of evidence of previously immunized persons being reinfected (although the titers may be low after immunization, in the event of an exposure to HBV, the body will show a protective immune response). As of today, most dental institutions in India require dental students and faculty to complete a series of vaccinations as a protection against this disease. Vaccination must be mandated for all oral health professionals if they have not been protected. Vaccines can be unique to HBV or are available as a combination of both HAV and HBV. Vaccination policy must be mandated for oral health personnel and must be enforced as it is the first and the best line of defense.

Hepatitis C virus (HCV), the parentally transmitted non-A non$B$ virus is an RNA virus, usually seen in association with blood transfusions and contact with blood and other body fluids. ${ }^{17}$ This disease can be very debilitating and fatal. Over $60 \%$ of the infected may develop chronic liver disease. Of those who develop liver disease, 30 to $60 \%$ develop active liver disease and 5 to $20 \%$ cirrhosis of the liver.

HCV infection and hepatocellular carcinoma are found to be epidemiologically associated. Although a vaccine is not available, various treatments against this infection including chemotherapy, have shown to help in controlling the disease and reduce viral load. This virus is highly infective, therefore health care providers must take adequate precautions (universal/ standard precautions) while treating all patients.

Hepatitis $D$ viruses are a virus-like particle, always dependent on the presence of hepatitis $\mathrm{B}$ viral infection in the patient (piggyback virus). It may occur as a coinfection with $\mathrm{HBV}$ or after being infected by HBV. Mode of transmission is similar to blood and other body fluid contact. ${ }^{18,19}$

Hepatitis $G$ viral infection is the most recent type that has been identified. It is a bloodborne condition.

In short, hepatitis viral infections are the most insidious infections that occur among susceptible patients. Dentists must avoid contact with blood and other body fluids of patients by using adequate barrier techniques, and adopting safe practices for the disposal of waste to avoid cross-infection among other patients. ${ }^{10}$ Further, health policy must address immunization if vaccines are available for these conditions as the first line of defense and postexposure protocols must consider these diseases if testing is available.

Human immunodeficiency virus (HIV) is a condition where transmission occurs through contact with blood and other body fluids. This disease was identified in June 1981, and has been the plague of the 20th century. Initially, it was seen among homosexual individuals and later found its way into all segments of society, including heterosexuals, females and children. This infection is on the rise in South Asia and Southeast Asia, 9,20 while in the US, infection rates are on the decline or have been stable. An initial HIV infection progresses into a more severe and debilitating condition where it is associated with a variety of other infections and is called AIDS or acquired immunodeficiency syndrome.

There are many classifications for AIDS, such as the Center for Disease Control's surveillance definition, the Walter Reed's classification or the WHO's classification. In early stages, the HIV infection may not be noticeable, and may be accompanied by symptoms, such as weakness, arthralgias, or even be totally asymptomatic. Upon progression, HIV infection may be associated with a variety of conditions. Some of the oral lesions associated with HIV infection and AIDS are hairy leukoplakia, Kaposi's sarcoma and candidiasis. It is imperative that dentists have knowledge of the clinical appearance of these oral lesions.

In addition to the oral conditions, there may be systemic conditions, such as protozoal infections, fungal infections, other viral infections and mycobacterial infections. Almost each of the organ systems may be involved in this infectious process as well. 
Although, there were patients infected by a dentist in Florida (USA), no other cases of transmission from a dentist have been reported in the US. There have been no occupational exposures leading to HIV infection of the dentist or dental auxiliaries during dental treatment in the US. It is absolutely essential to understand that in order to reduce the probability of seroconversion, postexposure protocols must be followed, and this means taking antiviral drugs immediately after exposure to a patient infected with HIV (within 2 hours after exposure to infected blood).

Tuberculosis is one of the oldest infectious diseases known to humans. In the past, most countries had TB under control. But now, it has re-emerged in both prevalence and with a new type of a multidrug-resistant strain. Mycobacterium tuberculosis is the organism that commonly affects the lungs but may involve most organs in the body.

Each year, about 8 million people develop TB and 3 million die. TB mimics many respiratory conditions, therefore when the practitioner observes a cough for more than 3 weeks of duration and sputum possibly tinged with blood, the patient should be referred for a TB skin test, and if diagnosed with active infection, treatment prescribed. It is pragmatic to defer care for patients with active TB until the disease is controlled. ${ }^{21,22}$

In the United States, dentists can defer elective dental care until the patient is pronounced noninfectious, and all emergency dental treatments may be provided in institutions that are equipped to deal with the control of cross-contamination or occupational exposure. Such facilities should include negative air pressure treatment rooms with the air vented to the outside of the building. The air-conditioning and ventilation system must also be equipped with HEPA (high efficiency particulate air) filters, and during contact with infected patients personnel must use masks that have a HEPA filter.

Dentists and staff must undergo testing for the disease on a periodic basis, especially if living in endemic areas where the prevalence is high (every 6 months in high endemic areas, and once yearly in moderate endemic areas). Many health care institutions in the United States have made annual TB testing mandatory for their personnel and have effective TB control plans. Similarly, public health policy should dictate that all oral health personnel in India must undergo testing for TB at least annually. If found positive (other than due to BCG vaccine), they must undergo treatment till pronounced noninfectious.

Although, the incidence of TB may not be increasing, diagnosis of multidrug-resistant TB is increasing in India. ${ }^{23}$ Mortality rate of MDR-TB in India is high and needs to be addressed. Drug resistance can be defined as "decrease in sensitivity to a sufficient degree to be reasonably certain that the strain of the mycobacterium in question concerned is different from a sample of wild strains of human type that have never come in contact with the drug". ${ }^{24}$ According to the consensus statement of the Indian Council of Medical Research (2007), ${ }^{25}$ MDR-TB is defined as "resistance to Isoniazid and Rifampicin with or without resistance to other anti-TB drugs".
Extensively drug resistance tuberculosis (XDR-TB) is defined as "resistance to atleast Isoniazid and Rifampicin (i.e. MDRTB) plus resistance to any of the Fluoroquinolones and any one of the second-line injectable drugs (Amikacin, Kanamycin, or Capreomycin)."

XDR-TB is on the rise in India. According to India's health ministry records, about $3 \%$ of the newly diagnosed cases have XDR-TB, and about $12 \%$ of previously diagnosed TB cases that are being treated have converted to this strain. ${ }^{26}$ These cases of XDR-TB are normally associated with patients that have HIV/AIDS and are very difficult to treat or may not respond to even newer generation of antituberculosis treatment regimen. According to the Director General of the Indian TB Control Program, there is an incidence of about 1.8 million cases in India, of which 0.8 million are deemed infectious. If the patients are not treated effectively, each patient can develop incurable forms of TB and infect 10 to 15 people a year. No new drugs have been discovered since 1968, the germs have now developed drug resistance. ${ }^{26}$

Severe acute respiratory syndrome is a coronavirus associated respiratory infection that was detected in 2003 and became a pandemic affecting over two-dozen countries (Fig. 1). ${ }^{27,28}$ It is currently contained and referred to as SARS-CoV. In the 2003 outbreaks, it affected more than 8000 people with a mortality rate close to $10 \%$ among infected. Very few cases and deaths were seen in medically advanced countries. The signs and symptoms include body temperature of $>100.4^{\circ} \mathrm{F}$, headache, bodyache with mild respiratory discomfort at the outset followed by moderate to severe respiratory distress, dry cough and many developing pneumonia. Around 10 to $20 \%$ have diarrhea. The SARS spreads through close contact, fomites through droplet infection (droplets can travel up to 3 feet) and through aerosolization. Close contact can include sharing spaces as in a family or at work where people spend a significant amount of time together. A clinic waiting room does not normally represent a place of close contact due to the amount of time spent together is minimal. Methods of control of SARS in a dental clinic would be to let patients know that they should not come to the clinic for a scheduled nonemergent appointment if they are not feeling well. Schedule them for another day when the patient is feeling better. Cover face while sneezing with a disposable tissue or the sleeve of your shirt/coat. Wash hands frequently and regularly, use alcohol based hand gels or hand sprays and wipe with disposable hand wipes if washing is not possible. Wear facemasks regularly to control inhalation of droplets (but will not stop breathing of aerosols).

AH1N1 influenza is a virus of swine origin with hosts being pigs and humans (Fig. 2). ${ }^{29}$ Etiology is coming into contact with an infected pig or human. Modes of transmission are through body secretions/fluids, droplets, aerosols and fomites. The incubation period is about 1 to 7 days and could be as little as 4 days. Transmission can occur from pigs to humans and from humans to humans. Signs and symptoms like cough, sore throat, rhinorrhea, acute febrile respiratory illness, headache, 


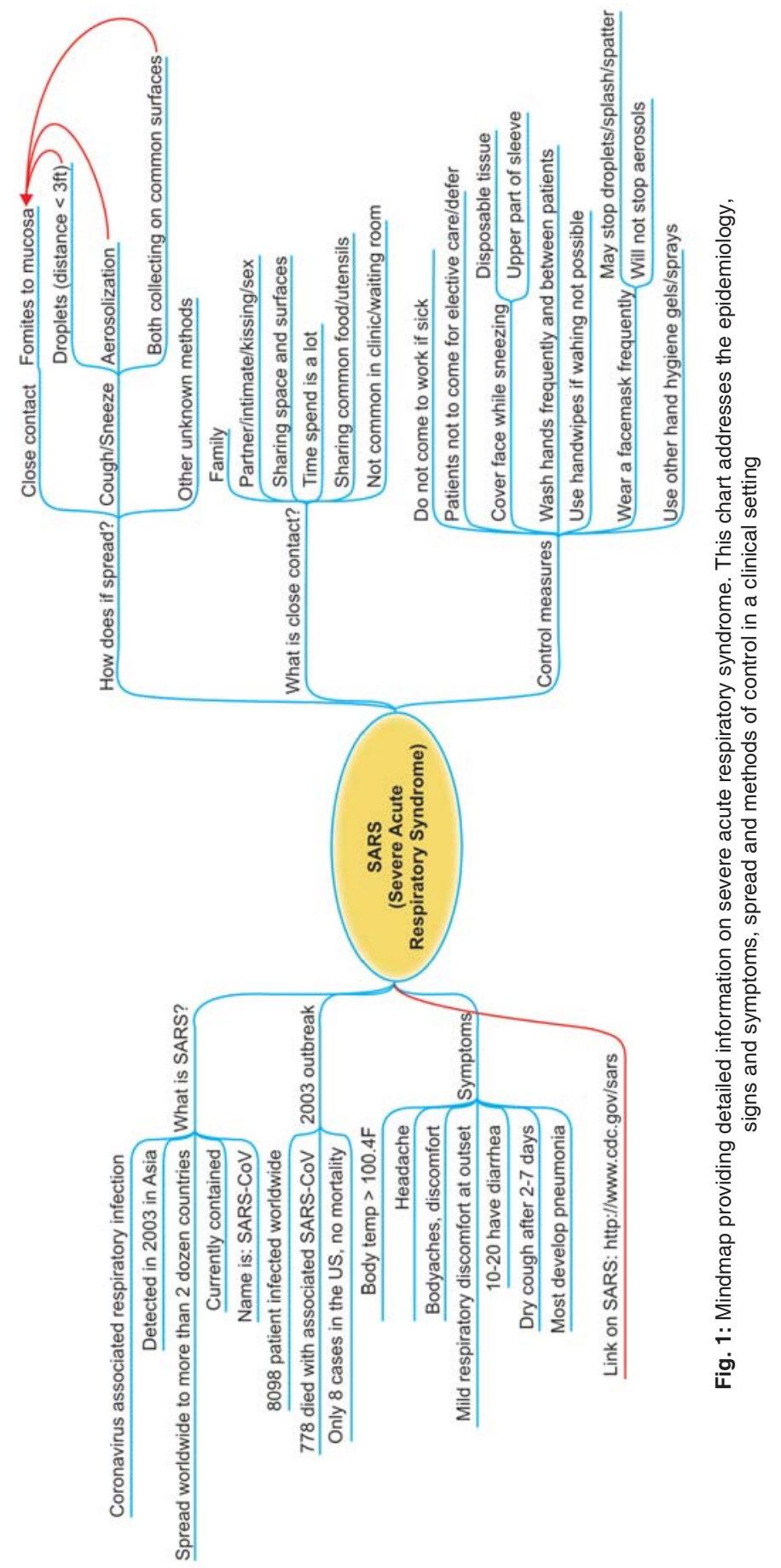




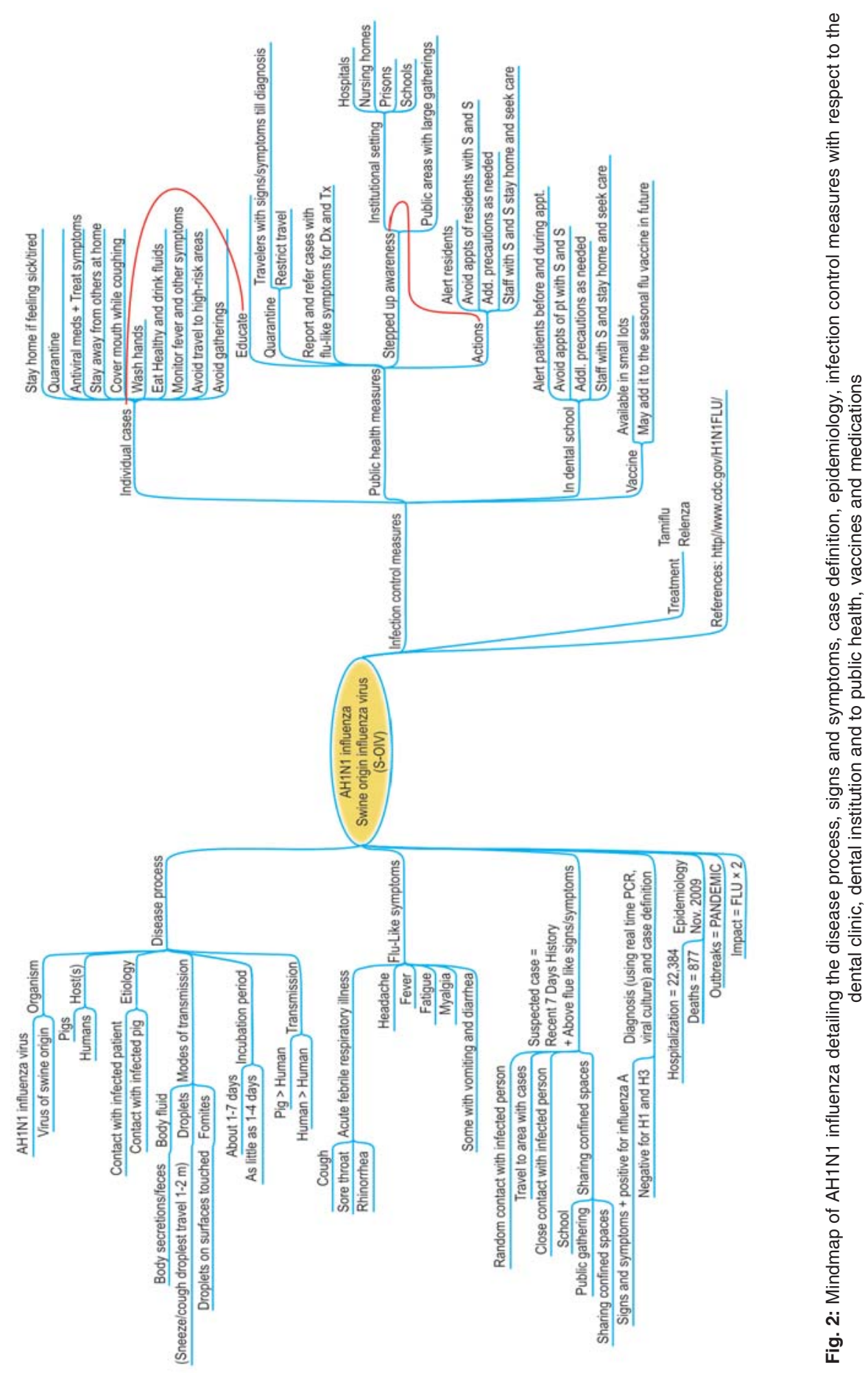


fever, fatigue, myalgia, vomiting and diarrhea are also not uncommon. A suspected case of AH1N1 influenza is recent history of exposure in addition to the signs and symptoms mentioned earlier (coming in contact with infected person, travel to areas with diagnosed cases, close contact with an infected patient, being in a school or institution or public gathering or sharing confined spaces where one could come in contact with a case). Diagnosis is use of a real-time polymerase chain reaction (PCR), viral culture and having signs and symptoms and being positive for influenza A and negative for $\mathrm{H} 1$ and $\mathrm{H} 3$. The epidemiology, as recorded in November 2009, demonstrated that there were 22,364 hospitalizations with about 877 deaths. These outbreaks were classified as a pandemic and was twice as severe in number as seasonal flu. Treatment of active infection is by using Tamiflu or Relenza, and it is advisable to get vaccinated. Today, a regular seasonal flu vaccine also has the components/vaccine to combat AH1N1 influenza. Common control methods in a dental clinic would be to let patients know that they should not come to the clinic for a scheduled nonemergent appointment if they are not feeling well. Schedule them for another day when the patient is feeling better. Cover face while sneezing with a disposable tissue or the sleeve of your shirt/coat. Wash hands frequently and regularly, use alcohol based hand gels or hand sprays and wipe with disposable hand wipes if washing is not possible. Wear facemasks regularly to control inhalation of droplets (but will not stop breathing of aerosols). Avoid going to public gatherings and use additional precautions as needed.

Childhood diseases that have vaccines available and commonly encountered are chickenpox, measles, mumps, rubella, diphtheria, pertussis, tetanus, rubeola, meningitis and poliomyelitis. All health professionals including dental students must utilize these vaccines. Further, to be admitted into dental/ health profession schools, it must be mandated that applicants show proof of immunization or undergo immunization before admission. $^{30,31}$

In India, all the dental schools, the dental council and other public health organizations must work together in developing consensus on immunization of health professionals and immunization for students applying to careers in health professions. Further, all residents of dormitories, student lodging and other residential institutions must implement immunization requirement for all residents.

In conclusion, basic knowledge of common infectious diseases is essential to control spread of infectious diseases. Immunization is the first line of defense against most common diseases and must be encouraged in health professions.

\section{REFERENCES}

1. Runnells R. Infectious diseases important in dentistry. Practical infection control in dentistry. Cottone J, Terezhalmy G, Molinari J (Eds). Philadelphia: Lea and Febiger 1991:1-17.

2. Puttaiah R, Cooley R. Infection control and personnel safety in periodontics. Fundamentals of periodontics. Wilson T, Kornman K (Eds). Chicago: Quintessence Publishing C, Inc 1996: 179-94.
3. Puttaiah R, Cooley R. Infection control and safety in orthodontics. Orthodontics for the next millenium. Sachdeva R, Bartleon HR, White L, Johnson J (Eds). Glendora: ORMCO 1997:519-40.

4. Benenson AS. Viral hepatitis. Control of communicable diseases manual-an official report of the American Public Health Association (16th edn). Washington DC. APHA 1995:217-33.

5. Cottone JA, Puttaiah R. Viral hepatitis and hepatitis vaccines. Practical infection control in dentistry (2nd edn). Cottone JA, Terezhalmy GT, Molinari JA(Eds). Philadelphia: Williams and Wilkins 1995:15-47.

6. Cottone JA, Puttaiah R. Hepatitis B virus infection current status in dentistry. Dental clinics of North America. April 1996;40(2).

7. Benenson AS. Tuberculosis. Control of communicable diseases manual: An official report of the American Public Health Association (16th ed), Washington DC: APHA 1995: 488-99.

8. Yeh CK, Puttaiah R, Prows J. HIV and AIDS. Practical infection control in dentistry (2nd ed). Cottone JA, Terezhalmy GT, Molinari JA (Eds). Philadelphia: Williams and Wilkins 1995: 48-81.

9. UNAIDS report on the global AIDS epidemic 2010: Global report: UNAIDS report on the global AIDS epidemic 2010. “UNAIDS/10.11E JC1958E” ISBN 978-92-9173-871-7. http:/ /www.unaids.org/globalreport/default.htm.

10. Centers for disease control: Update: Universal precautions of prevention of transmission of HIV, HBV and other bloodborne pathogens in health-care settings. MMWR 1988;37:377.

11. Multidrug and extensively drug-resistant TB (M/XDR-TB). 2010 Global report on surveillance and response. WHO/HTM/TB/ 2010.3. ISBN 978924159919 1. http://whqlibdoc.who.int/ publications/2010/9789241599191_eng.pdf.

12. Public health guidance for community-Level preparedness and response to severe acute respiratory syndrome (SARS). The centers for disease control and prevention. 08 Jan, 2004. http:// www.cdc.gov/ncidod/sars/guidance/core/pdf/core.pdf.

13. The 2009 H1N1 pandemic: Summary highlights April 2009April 2010. The centers for disease control and prevention H1N1 flu general information. http://www.cdc.gov/h1n1flu/ cdcresponse.htm.

14. Jacobsen KH. The global prevalence of hepatitis A virus infection and susceptibility: A systematic review. Geneva, Switzerland: World Health Organization 2009. http://whqlibdoc.who.int/hq/ 2010/WHO_IVB_10.01_eng.pdf

15. Hepatitis E. WHO department of communicable disease surveillance and response. WHO/CDS/CSR/EDC/2001.12. 1-28. http://www.who.int/csr/disease/hepatitis/HepatitisE_ whocdscsredc2001_12.pdf

16. Hepatitis B. WHO department of communicable disease surveillance and response. WHO/CDS/CSR/EDC/2002.2. Hepatitis B:1-76. http:/www.who.int/csr/disease/hepatitis/ HepatitisB_whocdscsrlyo2002_2.pdf

17. Hepatitis $\mathrm{C}$ virus. Department of communicable disease surveillance and response. WHO/CDS/CSR/LYO/2003:1-69. http://www.who.int/csr/disease/hepatitis/Hepc.pdf

18. Taylor JM. “Hepatitis delta virus”. Virology (1):71-76, January 2006.

19. Radjef N, Gordien E, Ivaniushina V, et al. Molecular phylogenetic analyses indicate a wide and ancient radiation of African hepatitis delta virus, suggesting a delta virus genus of at least seven major clades. J Virol March 2004;78(5):2537-44.

20. UNAIDS/06.20E. Report on the Global AIDS Epidemic: Executive Summary-A UNAIDS 10 anniversary special 
edition. WHO Library Cataloguing-in-Publication Data. "ISBN 929173511 6” UNAIDS20 avenue Appia, CH-1211, Geneva, Switzerland. Web site: www.unaids.org 2006.

21. Centers for disease control: Guideline for preventing the transmission of mycobacterium tuberculosis in health-care facilities, Fed Reg 1994;59:54242-303.

22. WHO: The global plan to stop TB 2011-2015: Transforming the fight towards elimination of tuberculosis. ISBN 978924 150034 0. http://www.stoptb.org/assets/documents/global/plan/ TB_GlobalPlanToStopTB2011-2015.pdf

23. Paramasivan CN, Venkataraman P. Drug resistance in tuberculosis in India. Indian J Med Res 120, October 2004;37786. http://medind.nic.in/iby/t04/i10/ibyt04i10p377.pdf

24. Mitchison DA. Drug resistance in mycobacteria. Br Med Bull 1984;40:84-90.

25. Multi-drug resistant and extensively drug resistant TB in India. Consensus statement on the problem, prevention, management and control. From the consultative meeting of national experts organized by the TB Research Centre, ICMR, Govt. of India, on 14-15 September 2007, at Chennai. http://www.tbcindia.org/ pdfs/Consensus\%20statement\% 20on\%20MDR\%20XDR\% 20TB\%20-Final.pdf
26. XDR-TB - Danger Ahead. News and Notes. Indian J Tuberc 2006;53:234.

27. Department of health and human services. The centers for disease control and prevention. Severe acute respiratory syndrome (SARS). SARS Home. http://www.cdc.gov/ncidod/sars/ index.htm

28. Cauchemez S, et al. Real time estimates in the early detection of SARS. Emerging infectious diseases. www.cdc.gov/eid 12(1), January 2006. http://www.cdc.gov/ncidod/EID/vol12no01/pdfs/ 05-0593.pdf

29. Department of health and human services. The centers for disease control and prevention. The 2009 H1N1 pandemic: Summary highlights, April 2009-April 2010. http://www.cdc.gov/h1n1flu/ cdcresponse.htm

30. Immunization of health-care workers: Recommendations of the advisory committee on immunization practices (ACIP) and the hospital infection control practices advisory committee (HICPAC). Recommendations and reports, MMWR, December 26,1997/46(RR-18);1-42.

31. Yale Health, Yale University, New Haven, CT, USA. http:// yalehealth.yale.edu/med_services/immunization-faq.html 


\title{
Advertising \& Marketing
}

\section{Dedicated to Mark Your Presence in Medical Fraternity}

\author{
We Extend Our Effectual Support in Form of:
}

\section{Advertisement | Bulk Subscription | Reprints | e-Prints Online Advertisement | Customization | CME | Endorsements}

- Our tactical and conversant publishing team owns a targeted approach to aid you in designing customized market solutions to make you reach key markets and also enjoy business proliferation with our competitive pricing.

- Being world's leading, extensive medical publishers, our state-of-art products and services developed under the close association of local and international medical experts meet your marketing and educational program requirements productively.

- Our growing international associations give you a platform to experience campaign on global front.

- Our PER division includes a team of renowned doctors and specialists to undertake content management foryour products and services.

\section{Explore more at:}

www.jaypeebrothers.com / www.jaypeejournals.com

(11) Jaypee Brothers Medical Publishers Pvt. Ltd.

\section{Domestic \& Overseas Operations}

Ahmedabad • Bengaluru $\bullet$ Chennai $\bullet$ Hyderabad $\bullet$ Kochi $\bullet$ Kolkata $\bullet$ Lucknow $\bullet$ Mumbai $\bullet$ Nagpur

- London (UK) • Panama City (Panama) • St Louis (USA)

\section{Corporate Office}

4838/24 Ansari Road, Daryaganj, NewDelhi 110 002, India

Phone: 91-11-43574357

e-mail: jaypee@jaypeebrothers.com 